MATHEMATICS OF COMPUTATION

Volume 73, Number 247, Pages 1365-1384

S 0025-5718(03)01622-3

Article electronically published on December 19, 2003

\title{
CONVEXITY RESULTS AND SHARP ERROR ESTIMATES IN APPROXIMATE MULTIVARIATE INTEGRATION
}

\author{
ALLAL GUESSAB AND GERHARD SCHMEISSER
}

\begin{abstract}
An interesting property of the midpoint rule and the trapezoidal rule, which is expressed by the so-called Hermite-Hadamard inequalities, is that they provide one-sided approximations to the integral of a convex function. We establish multivariate analogues of the Hermite-Hadamard inequalities and obtain access to multivariate integration formulae via convexity, in analogy to the univariate case. In particular, for simplices of arbitrary dimension, we present two families of integration formulae which both contain a multivariate analogue of the midpoint rule and the trapezoidal rule as boundary cases. The first family also includes a multivariate analogue of a Maclaurin formula and of the two-point Gaussian quadrature formula; the second family includes a multivariate analogue of a formula by P. C. Hammer and of Simpson's rule. In both families, we trace out those formulae which satisfy a Hermite-Hadamard inequality. As an immediate consequence of the latter, we obtain sharp error estimates for twice continuously differentiable functions.
\end{abstract}

\section{INTRODUCTION}

Let $f:[a, b] \rightarrow \mathbb{R}$ be a convex function. Then

$$
f\left(\frac{a+b}{2}\right) \leq \frac{1}{b-a} \int_{a}^{b} f(x) \mathrm{d} x \leq \frac{1}{2}(f(a)+f(b)) .
$$

These inequalities are of fundamental interest in numerical quadrature. They say that the midpoint rule approximates the integral from below and the trapezoidal rule approximates it from above. Moreover, these inequalities have attracted the interest of people working in general inequalities, who refer to (1.1) as the HermiteHadamard inequalities; see [6]. We shall join them in doing so. In particular, we shall call the first and the second inequality in (1.1) the lower and the upper Hermite-Hadamard inequality, respectively.

The midpoint rule and the trapezoidal rule are the simplest quadrature formulae. They can serve as basic elements for constructing more sophisticated formulae by certain types of convex combinations or for deducing various quadrature procedures by subdivisions and compositions (see [14] and [15] $\S 3.2,4.2]$, where efficient algorithms for various types of improper integrals are obtained). For these reasons, the midpoint rule and the trapezoidal rule together with their fundamental inequality (1.1) have been a starting point for numerous investigations.

Received by the editor October 24, 2002.

2000 Mathematics Subject Classification. Primary 65D30, 65D32, 41A63, 41A44, 41A80; Secondary 26B25, 26D15, 52A40.

Key words and phrases. Multivariate approximate integration, convex functions, HermiteHadamard inequality, error estimates. 
For example, we may consider a convex combination of the nodes of the two formulae by defining

$$
\begin{aligned}
& x_{1}(\lambda):=\lambda a+(1-\lambda) \frac{a+b}{2}=\frac{1+\lambda}{2} a+\frac{1-\lambda}{2} b, \\
& x_{2}(\lambda):=\lambda b+(1-\lambda) \frac{a+b}{2}=\frac{1-\lambda}{2} a+\frac{1+\lambda}{2} b .
\end{aligned}
$$

Then

$$
\frac{1}{b-a} \int_{a}^{b} f(x) \mathrm{d} x=\frac{1}{2}\left[f\left(x_{1}(\lambda)\right)+f\left(x_{2}(\lambda)\right)\right]+R(\lambda)[f], \quad \lambda \in[0,1],
$$

is a family of quadrature formulae which gives the midpoint rule when $\lambda=0$ and the trapezoidal rule when $\lambda=1$. Moreover, for any convex function $f$, we have

$$
\frac{1}{2}\left[f\left(x_{1}(\lambda)\right)+f\left(x_{2}(\lambda)\right)\right] \leq \frac{1}{b-a} \int_{a}^{b} f(x) \mathrm{d} x \quad \text { for } 0 \leq \lambda \leq \frac{1}{2} ;
$$

see 8 . The extremal value $1 / 2$ of the parameter $\lambda$ gives the two-point Maclaurin formula, not to be mistaken for the Euler-Maclaurin formula. For $\lambda=3^{-1 / 2}$, the formula (1.2) yields the two-point Gaussian quadrature formula, which is of order 4.

We may also consider a convex combination of the midpoint rule and the trapezoidal rule with fixed nodes, that is,

$$
\frac{1}{b-a} \int_{a}^{b} f(x) \mathrm{d} x=\alpha f\left(\frac{a+b}{2}\right)+\frac{1-\alpha}{2}(f(a)+f(b))+R^{*}(\alpha)[f], \quad \alpha \in[0,1] .
$$

This family gives the trapezoidal rule when $\alpha=0$ and the midpoint rule when $\alpha=1$. Moreover, for any convex function $f$, we have

$$
\frac{1}{b-a} \int_{a}^{b} f(x) \mathrm{d} x \leq \alpha f\left(\frac{a+b}{2}\right)+\frac{1-\alpha}{2}(f(a)+f(b)) \quad \text { for } 0 \leq \alpha \leq \frac{1}{2} .
$$

The extremal value $1 / 2$ of the parameter $\alpha$ gives an inequality which was discovered by Hammer [9], but sometimes it is also attributed to Bullen [3]; see [6] p. 11]. For $\alpha=2 / 3$, the formula (1.4) yields Simpson's rule, which is of order 4 .

In this paper, we look for multivariate analogues of these classical results. As regards convexity, it was mentioned in [12, $\S 8 \mathrm{~d}]$ that not much has been done in multivariate integration.

For any integration formula, we shall say that it admits a lower or an upper Hermite-Hadamard inequality if it approximates the integral of any convex function from below or above, respectively. We shall see that for such an integration formula we can always establish a sharp error bound.

By an affine function $L$ on $\mathbb{R}^{d}$ we mean a mapping

$$
L: \boldsymbol{x} \longmapsto a+\langle\boldsymbol{b}, \boldsymbol{x}\rangle,
$$

where $a \in \mathbb{R}, \boldsymbol{b}, \boldsymbol{x} \in \mathbb{R}^{d}$, and $\langle\boldsymbol{b}, \boldsymbol{x}\rangle$ is the standard inner product of $\boldsymbol{b}$ and $\boldsymbol{x}$. If $a=0$, then the function $L$ is linear. Since, for an affine function $L$, both $L$ and $-L$ are convex, it follows that if an integration formula admits a (lower or upper) Hermite-Hadamard inequality, then it must be exact for affine functions. 


\section{Multivariate analogues}

OF THE MIDPOINT AND THE TRAPEZOIDAL RULE

In view of (1.1), the midpoint rule and the trapezoidal rule can be characterized as the quadrature formulae with a minimal number of nodes such that for any convex function the integral is approximated from below and above, respectively. We shall use this characterization for locating multivariate analogues.

A property, which may be seen as a restriction in the multivariate case, is that convexity of a function requires the domain of definition to be convex. For example, it may happen that a globally convex function has to be integrated over a nonconvex domain $\mathcal{D}$. Then the rich theory on convex functions would not apply to $f$ restricted to $\mathcal{D}$. However, various attempts have been made in order to define convex functions on non-convex domains. One of the most useful concepts is that of Busemann and Shephard [4], which is as follows.

Definition (Busemann-Shephard). Let $\mathcal{D} \subseteq \mathbb{R}^{d}$. A function $f: \mathcal{D} \rightarrow \mathbb{R}$ is called convex on $\mathcal{D}$ if it has the following properties:

(i) whenever $k \in \mathbb{N}, \boldsymbol{x}_{j} \in \mathcal{D}$, and $\lambda_{j} \in[0,1]$ for $j=1, \ldots, k$ such that

$$
\sum_{j=1}^{k} \lambda_{j}=1 \quad \text { and } \quad \sum_{j=1}^{k} \lambda_{j} \boldsymbol{x}_{j} \in \mathcal{D},
$$

then

$$
f\left(\sum_{j=1}^{k} \lambda_{j} \boldsymbol{x}_{j}\right) \leq \sum_{j=1}^{k} \lambda_{j} f\left(\boldsymbol{x}_{j}\right)
$$

(ii) there exists an affine function $L$ such that $L(\boldsymbol{x}) \leq f(\boldsymbol{x})$ for all $\boldsymbol{x} \in \mathcal{D}$.

It follows that if $\mathcal{D}$ is a convex set, then $f$ is convex in the sense of BusemannShephard if and only if it is convex in the classical sense. Moreover, if $f$ is convex in the sense of Busemann-Shephard, then it has an extension $\tilde{f}$ to the convex hull of $\mathcal{D}$ where $\tilde{f}$ is convex in the classical sense. Conversely, if a convex function is restricted to a non-convex subset of its domain of definition, then the restriction is convex in the sense of Busemann-Shephard.

An important property of the above notion is that it guarantees the existence of supporting hyperplanes.

Proposition A [4 p. 21]. Let $f$ be convex as in the above definition. Then, for each $\boldsymbol{y} \in \mathcal{D}$, there exists an affine function $L_{\boldsymbol{y}}$ such that

$$
f(\boldsymbol{y})=L_{\boldsymbol{y}}(\boldsymbol{y}) \quad \text { and } \quad f(\boldsymbol{x}) \geq L_{\boldsymbol{y}}(\boldsymbol{x}) \quad \text { for all } \boldsymbol{x} \in \mathcal{D} .
$$

For a measurable set $\mathcal{D} \subset \mathbb{R}^{d}$, we shall denote by $|\mathcal{D}|$ the measure of $\mathcal{D}$. The following simple theorem describes a multivariate analogue of the midpoint rule. That formula may be attributed to Good and Gaskins [7, but these authors did not present results for convex functions. In recent publications (see, e.g., 6, p. 257, Theorem 158, (6.33)] ), the authors did consider such a formula for convex functions, but they required differentiability and had restrictions on the domain of integration and the dimension $d$. 
Theorem 2.1. Let $\mathcal{D} \subset \mathbb{R}^{d}$ be a measurable set of positive measure, and suppose that $\mathcal{D}$ contains its center of gravity defined by

$$
\boldsymbol{x}^{*}:=\frac{1}{|\mathcal{D}|} \int_{\mathcal{D}} \boldsymbol{x} \mathrm{d} \boldsymbol{x} .
$$

Let $f$ be convex on $\mathcal{D}$ in the sense of Busemann-Shephard. Then

$$
f\left(\boldsymbol{x}^{*}\right) \leq \frac{1}{|\mathcal{D}|} \int_{\mathcal{D}} f(\boldsymbol{x}) \mathrm{d} \boldsymbol{x} .
$$

Equality is attained for every affine function $f$. Moreover, in (2.1), the point $\boldsymbol{x}^{*}$ cannot be replaced by any other $\boldsymbol{y} \in \mathcal{D}$.

We want to call the integration formula

$$
\frac{1}{|\mathcal{D}|} \int_{\mathcal{D}} f(\boldsymbol{x}) \mathrm{d} \boldsymbol{x}=f\left(\boldsymbol{x}^{*}\right)+R_{d}^{\mathrm{cg}}[f]
$$

the center-of-gravity rule. For a connected set $\mathcal{D} \subset \mathbb{R}$, it reduces to the midpoint rule.

We now turn to a generalization of the trapezoidal rule. As regards general domains of integration, we will be much more modest, but subsequently we shall see that we cannot do more.

Theorem 2.2. Let $\mathcal{S} \subset \mathbb{R}^{d}$ be a non-degenerate simplex with vertices $\boldsymbol{x}_{0}, \ldots, \boldsymbol{x}_{d}$, and let $f$ be convex on $\mathcal{S}$. Then

$$
\frac{1}{|\mathcal{S}|} \int_{\mathcal{S}} f(\boldsymbol{x}) \mathrm{d} \boldsymbol{x} \leq \frac{1}{d+1} \sum_{i=0}^{d} f\left(\boldsymbol{x}_{i}\right) .
$$

Equality is attained if and only if $f$ is an affine function.

We want to call the integration formula

$$
\frac{1}{|\mathcal{S}|} \int_{\mathcal{S}} f(\boldsymbol{x}) \mathrm{d} \boldsymbol{x}=\frac{1}{d+1} \sum_{i=0}^{d} f\left(\boldsymbol{x}_{i}\right)+R_{d}^{\mathrm{vs}}[f]
$$

the vertices-of-a-simplex rule. For a non-degenerate simplex in $\mathbb{R}$, it reduces to the trapezoidal rule. For $d=2$ the simplices are triangles, and then the inequality (2.3) was proved by Allasia and Giordano [1] who considered their integration formula as a two-dimensional analogue of the trapezoidal rule. Formula (2.4) in its full generality is contained in the book of Stroud [16, p. 307, formula $\left.T_{n}: 1-2\right]$ where it is attributed to $\mathrm{R}$. Lauffer.

There is a big discrepancy between the admissible domains of integration in Theorems 2.1 and 2.2. But actually, in Theorem 2.2, we cannot admit domains other than simplices if we want a minimal number of nodes.

Theorem 2.3. Let $\mathcal{K} \subset \mathbb{R}^{d}$ be a convex set of finite positive measure. Suppose that there are points $\boldsymbol{x}_{0}, \ldots, \boldsymbol{x}_{n} \in \mathcal{K}$ and real numbers $A_{0}, \ldots, A_{n}$ such that, for every convex function $f$ on $\mathcal{K}$, we have

$$
\frac{1}{|\mathcal{K}|} \int_{\mathcal{K}} f(\boldsymbol{x}) \mathrm{d} \boldsymbol{x} \leq \sum_{\nu=0}^{n} A_{\nu} f\left(\boldsymbol{x}_{\nu}\right)
$$

Then the following holds:

(i) $\mathcal{K}$ is the convex hull of $\boldsymbol{x}_{0}, \ldots, \boldsymbol{x}_{n}$; 
(ii) $n \geq d$;

(iii) if $\boldsymbol{x}_{j}$ is an extremal point of $\mathcal{K}$, then $A_{j}>0$;

(iv) if $n=d$, then the integration formula on the right-hand side of (2.5) is the vertices-of-a-simplex rule.

Theorem 2.3 shows that an upper Hermite-Hadamard inequality can only exist on convex polytopes. Such an inequality can be established by decomposing the convex polytope into simplices whose vertices are amongst the vertices of the polytope. Then (2.3) holds on each simplex and extends to the polytope by combining the resulting inequalities. The decomposition of a convex polytope into simplices is not unique. We may therefore consider all possible decompositions and take the arithmetic mean of all the resulting integration formulae. Then, for a regular polytope $\mathcal{P}$, we obtain an integration formula all whose coefficients are equal. If, in this case, $\mathcal{P}$ has $k$ vertices $\boldsymbol{x}_{1}, \ldots, \boldsymbol{x}_{k}$, then the formula is

$$
\frac{1}{|\mathcal{P}|} \int_{\mathcal{P}} f(\boldsymbol{x}) \mathrm{d} \boldsymbol{x}=\frac{1}{k} \sum_{i=1}^{k} f\left(\boldsymbol{x}_{i}\right)+R_{d}^{\mathrm{vp}}[f] .
$$

Theorem 2.3 shows, in particular, that there is no upper Hermite-Hadamard inequality for a disk. However, Dragomir [5] observed that, for a convex function, the integral mean over a disk is bounded by the integral mean over the circle which is the boundary of this disk. This he called a Hadamard inequality for the disk. His result can also be deduced by approximating the circle by a regular polygon with $k$ vertices, establishing a two-dimensional Hermite-Hadamard inequality for the resulting polygonal domain, and letting $k$ tend to infinity.

We have seen that the center-of-gravity rule and the vertices-of-a-simplex rule give Hermite-Hadamard inequalities with a minimal number of nodes. However, there is a price to pay for this minimality. In the case of a simplex, we can show that amongst all integration formulae, which have positive coefficients and approximate the integral of a convex function from below or above, the center-of-gravity rule and the vertices-of-a-simplex rule, respectively, yield the worst approximation.

Theorem 2.4. Let $\mathcal{S} \subset \mathbb{R}^{d}$ be a non-degenerate simplex with vertices $\boldsymbol{x}_{0}, \ldots, \boldsymbol{x}_{d}$ and center of gravity $\boldsymbol{x}^{*}$. Suppose that there are points

$$
\boldsymbol{y}_{0}, \ldots, \boldsymbol{y}_{m}, \boldsymbol{z}_{0}, \ldots, \boldsymbol{z}_{n} \in \mathcal{S}
$$

and positive numbers $A_{0}, \ldots, A_{m}, B_{0}, \ldots, B_{n}$ such that, for every convex function $f$, we have

$$
\sum_{\mu=0}^{m} A_{\mu} f\left(\boldsymbol{y}_{\mu}\right) \leq \frac{1}{|\mathcal{S}|} \int_{\mathcal{S}} f(\boldsymbol{x}) \mathrm{d} \boldsymbol{x} \leq \sum_{\nu=0}^{n} B_{\nu} f\left(\boldsymbol{z}_{\nu}\right)
$$

Then

$$
f\left(\boldsymbol{x}^{*}\right) \leq \sum_{\mu=0}^{m} A_{\mu} f\left(\boldsymbol{y}_{\mu}\right)
$$

and

$$
\sum_{\nu=0}^{n} B_{\nu} f\left(\boldsymbol{z}_{\nu}\right) \leq \frac{1}{d+1} \sum_{i=0}^{d} f\left(\boldsymbol{x}_{i}\right)
$$




\section{A multivariate analogue of a Maclaurin formula}

The Maclaurin formula mentioned in the introduction can be characterized as the quadrature formula with the following properties:

(i) Its nodes are a convex combination of the nodes of the midpoint and the trapezoidal rule.

(ii) It admits a lower Hermite-Hadamard inequality.

(iii) Amongst all formulae satisfying (i) and (ii), it is the one which yields the best approximation to the integral of a convex function.

These properties may be used for tracing out a multivariate analogue of the two-point Maclaurin formula.

Considering a non-degenerate simplex $\mathcal{S} \subset \mathbb{R}^{d}$ with vertices $\boldsymbol{x}_{0}, \ldots, \boldsymbol{x}_{d}$ and center of gravity $\boldsymbol{x}^{*}$, we introduce

$$
\boldsymbol{x}_{i}(\lambda):=\lambda \boldsymbol{x}_{i}+(1-\lambda) \boldsymbol{x}^{*}, \quad 0 \leq \lambda \leq 1 .
$$

Then the following theorem holds.

Theorem 3.1. In the preceding notation, let $f$ be convex on $\mathcal{S}$. Then

$$
\frac{1}{d+1} \sum_{i=0}^{d} f\left(\boldsymbol{x}_{i}(\lambda)\right) \leq \frac{1}{|\mathcal{S}|} \int_{\mathcal{S}} f(\boldsymbol{x}) \mathrm{d} \boldsymbol{x}
$$

for $0 \leq \lambda \leq \lambda_{d}^{*}$, where

$$
\lambda_{d}^{*}=\frac{1}{d} \sum_{j=2}^{d+1} \frac{1}{j} .
$$

As a function of $\lambda$, the left-hand side of (3.2) is non-decreasing on [0,1]. Moreover, for every $\lambda \in\left(\lambda_{d}^{*}, 1\right]$, there exists a convex function $f$ for which (3.2) is false.

For $\lambda=\lambda_{d}^{*}$, the integration formula

$$
\frac{1}{|\mathcal{S}|} \int_{\mathcal{S}} f(\boldsymbol{x}) \mathrm{d} \boldsymbol{x}=\frac{1}{d+1} \sum_{i=0}^{d} f\left(\boldsymbol{x}_{i}(\lambda)\right)+R_{d}(\lambda)[f]
$$

may be called a multivariate Maclaurin formula. Since $\lambda_{1}^{*}=1 / 2$, we obtain the classical two-point Maclaurin formula when $d=1$.

Theorem 3.1 has an interesting consequence. For $\lambda=1$, the integration formula (3.4) is the vertices-of-a-simplex rule, which approximates the integral of a convex function from above (see Theorem 2.2). Hence, when $f$ is a continuous, convex function, then, by the intermediate-value theorem, there must exist a $\widetilde{\lambda} \in\left[\lambda_{d}^{*}, 1\right]$ such that the integration formula for $\widetilde{\lambda}$ gives the exact value of the integral. By a refined consideration, we shall see that we need not assume continuity. In fact, the following result holds.

Corollary 3.2. Let $\mathcal{S} \subset \mathbb{R}^{d}$ be a non-degenerate simplex with vertices $\boldsymbol{x}_{0}, \ldots, \boldsymbol{x}_{d}$. Suppose that $f$ is a convex function defined on $\mathcal{S}$. Then, in the above notation, there exists a $\widetilde{\lambda} \in\left[\lambda_{d}^{*}, 1\right)$ such that

$$
\frac{1}{|\mathcal{S}|} \int_{\mathcal{S}} f(\boldsymbol{x}) \mathrm{d} \boldsymbol{x}=\frac{1}{d+1} \sum_{i=0}^{d} f\left(\boldsymbol{x}_{i}(\widetilde{\lambda})\right) .
$$


In higher dimensions, this result is quite surprising for the following reason. Generally, for a continuous function $f$, we have

$$
\frac{1}{|\mathcal{S}|} \int_{\mathcal{S}} f(\boldsymbol{x}) \mathrm{d} \boldsymbol{x}=f(\widetilde{\boldsymbol{x}})
$$

for some $\widetilde{\boldsymbol{x}} \in \mathcal{S}$. Here $\widetilde{\boldsymbol{x}}$ may be anywhere in $\mathcal{S}$, depending on the function $f$. Since $\widetilde{\boldsymbol{x}}$ has $d$ components, we can say that we have $d$ "degrees of uncertainty". In the representation (3.5), we have nothing more than $\tilde{\lambda} \in\left[\lambda_{d}^{*}, 1\right.$ ). Hence, in any dimension $d$, we can represent the integral of a convex function with just one degree of uncertainty. Note that the points $\boldsymbol{x}_{0}(\lambda), \ldots, \boldsymbol{x}_{d}(\lambda)$ are from a very special and very "small" subset of $\mathcal{S}$, and they vary dependently as $\lambda$ moves in $\left[\lambda_{d}^{*}, 1\right]$.

\section{A multivariate analogue of Hammer's inequality}

The quadrature formula appearing in Hammer's inequality (the case $\alpha=1 / 2$ of (1.5) ) can be characterized as follows:

(i) It is a convex combination of the midpoint rule and the trapezoidal rule.

(ii) It admits an upper Hermite-Hadamard inequality.

(iii) Amongst all formulae satisfying (i) and (ii), it is the one which yields the best approximation to the integral of a convex function.

In view of this characterization, the following theorem allows us to locate a multivariate analogue of Hammer's result.

Theorem 4.1. Let $\mathcal{S} \subset \mathbb{R}^{d}$ be a non-degenerate simplex with vertices $\boldsymbol{x}_{0}, \ldots, \boldsymbol{x}_{d}$ and center of gravity $\boldsymbol{x}^{*}$. Then for every convex function $f: \mathcal{S} \rightarrow \mathbb{R}$ we have

$$
\frac{1}{|\mathcal{S}|} \int_{\mathcal{S}} f(\boldsymbol{x}) \mathrm{d} \boldsymbol{x} \leq \alpha f\left(\boldsymbol{x}^{*}\right)+(1-\alpha)\left[\frac{1}{d+1} \sum_{i=0}^{d} f\left(\boldsymbol{x}_{i}\right)\right]
$$

if $\alpha \in[0,1 /(d+1)]$. As a function of $\alpha$, the right-hand side of (4.1) is non-increasing on $[0,1]$. Moreover, for every $\alpha>1 /(d+1)$, there exists a convex function $f$ for which 4.1 is false.

For $\alpha=1 /(d+1)$, the integration formula

$$
\frac{1}{|\mathcal{S}|} \int_{\mathcal{S}} f(\boldsymbol{x}) \mathrm{d} \boldsymbol{x}=\alpha f\left(\boldsymbol{x}^{*}\right)+(1-\alpha)\left[\frac{1}{d+1} \sum_{i=0}^{d} f\left(\boldsymbol{x}_{i}\right)\right]+R_{d}^{*}(\alpha)[f]
$$

may be called the multivariate Hammer formula. For $d=1$, the corresponding $\alpha$ becomes $1 / 2$, and then we obtain the formula considered by Hammer 9 .

\section{A multivariate analogue of Simpson's Rule}

For $\alpha=(d+1) /(d+2)$, the family (4.2) yields a formula of order greater than 2. Indeed, the following theorem holds.

Theorem 5.1. For a non-degenerate simplex $\mathcal{S} \subset \mathbb{R}^{d}$ with vertices $\boldsymbol{x}_{0}, \ldots, \boldsymbol{x}_{d}$ and center of gravity $\boldsymbol{x}^{*}$, consider the integration formula

$$
\frac{1}{|\mathcal{S}|} \int_{\mathcal{S}} f(\boldsymbol{x}) \mathrm{d} \boldsymbol{x}=\frac{d+1}{d+2} f\left(\boldsymbol{x}^{*}\right)+\frac{1}{(d+2)(d+1)} \sum_{i=0}^{d} f\left(\boldsymbol{x}_{i}\right)+R_{d}^{\mathrm{Si}}[f] .
$$

Then $R_{d}^{\mathrm{Si}}[f]=0$ whenever $f$ is a polynomial in $d$ variables of total degree at most two. 
Obviously, for $d=1$, we obtain Simpson's rule. We may therefore call the integration formula (5.1) the multivariate Simpson rule for a simplex. It will turn out to be a useful tool for proving the results of the next section.

\section{ShaRP ERROR BOUNDS}

For a twice differentiable function $f: \mathcal{S} \rightarrow \mathbb{R}$ in $d$ variables, we denote by

$$
H[f](\boldsymbol{x}):=\left(\frac{\partial^{2} f}{\partial x_{i} \partial x_{j}}(\boldsymbol{x})\right)_{i, j=1, \ldots, d}
$$

the Hessian matrix of $f$ at $\boldsymbol{x}$, and we introduce

$$
\left|f^{\prime \prime}\right|:=\sup _{\boldsymbol{x} \in \mathcal{S}} \sup \left\{\left|\boldsymbol{y}^{\top} H[f](\boldsymbol{x}) \boldsymbol{y}\right|: \boldsymbol{y} \in \mathbb{R}^{d},\|\boldsymbol{y}\|=1\right\},
$$

where $\|\cdot\|$ is the Euclidean norm in $\mathbb{R}^{d}$ and $\boldsymbol{y}$ is assumed to be a column vector so that its transpose $\boldsymbol{y}^{\top}$ becomes a row vector.

An interesting and very useful property of Hermite-Hadamard inequalities is that they always yield a sharp error bound for the associated integration formula. This observation can be stated as follows.

Lemma 6.1. For a measurable set $\mathcal{D} \subset \mathbb{R}^{d}$ of positive measure, let

$$
\frac{1}{|\mathcal{D}|} \int_{\mathcal{D}} g(\boldsymbol{x}) \mathrm{d} \boldsymbol{x}=\sum_{\nu=0}^{n} A_{\nu} g\left(\boldsymbol{x}_{\nu}\right)+R_{d}[g]
$$

be an integration formula which admits an upper or a lower Hermite-Hadamard inequality. Then for every twice continuously differentiable function $f$ and any $\boldsymbol{y} \in \mathbb{R}^{d}$ we have

$$
\left|R_{d}[f]\right| \leq\left|R_{d}\left[\|\cdot-\boldsymbol{y}\|^{2}\right]\right| \cdot \frac{\left|f^{\prime \prime}\right|}{2} .
$$

This estimate is sharp. Equality is attained for every function of the form

$$
f(\boldsymbol{x}):=L(\boldsymbol{x})+c\|\boldsymbol{x}\|^{2},
$$

where $c \in \mathbb{R}$ and $L$ is any affine function.

In conjunction with Theorems 2.1 2.2, and 3.1, Lemma6.1]implies the following estimates.

Corollary 6.2. Let $\mathcal{S} \subset \mathbb{R}^{d}$ be a non-degenerate simplex with vertices $\boldsymbol{x}_{0}, \ldots, \boldsymbol{x}_{d}$ and center of gravity $\boldsymbol{x}^{*}$. Let $f: \mathcal{S} \rightarrow \mathbb{R}$ be twice continuously differentiable. Then, for the formulae (2.2), (2.4), and (3.4), the following error estimates hold:

$$
\begin{aligned}
\left|R_{d}^{\mathrm{cg}}[f]\right| & \leq \frac{\left|f^{\prime \prime}\right|}{2(d+1)(d+2)} \sum_{i=0}^{d}\left\|\boldsymbol{x}_{i}-\boldsymbol{x}^{*}\right\|^{2} ; \\
\left|R_{d}^{\mathrm{vs}}[f]\right| & \leq \frac{\left|f^{\prime \prime}\right|}{2(d+2)} \sum_{i=0}^{d}\left\|\boldsymbol{x}_{i}-\boldsymbol{x}^{*}\right\|^{2} ; \\
\left|R_{d}(\lambda)[f]\right| & \leq \frac{\left|f^{\prime \prime}\right|}{2(d+1)}\left(\frac{1}{d+2}-\lambda^{2}\right) \sum_{i=0}^{d}\left\|\boldsymbol{x}_{i}-\boldsymbol{x}^{*}\right\|^{2}
\end{aligned}
$$


for $0 \leq \lambda \leq \lambda_{d}^{*}$, where $\lambda_{d}^{*}$ is defined in (3.3). Each of these estimates is sharp. Equality is attained for every function of the form (6.3).

Let us mention that

$$
\sum_{i=0}^{d}\left\|\boldsymbol{x}_{i}-\boldsymbol{x}^{*}\right\|^{2}= \begin{cases}\frac{d^{2}}{d+1} & \text { for the standard unit simplex } \\ \frac{d}{2} & \text { for a regular simplex with edges of length } 1\end{cases}
$$

For $d=1$, Corollary 6.2 yields error estimates for the family (1.2). In particular, for $\lambda=1 / 2$, we obtain

$$
\left|R\left(\frac{1}{2}\right)[f]\right| \leq \frac{(b-a)^{2}}{96} \max _{a \leq x \leq b}\left|f^{\prime \prime}(x)\right|,
$$

which is a well-known estimate for the two-point Maclaurin formula; see for example [2, p. 128 , Satz $74, n=2, \beta=1 / 2$ ] or [10, p. 88 , No. 26].

Remark 6.3. For $\lambda=1 / \sqrt{d+2}$, the right-hand side of (6.6) would vanish, but this value of $\lambda$ is not admissible since it is larger than $\lambda_{d}^{*}$. Nevertheless, $\lambda=1 / \sqrt{d+2}$ is of interest since it is the only value of $\lambda$ for which the formula (3.4) is of order greater than 2. For $d=1$, the corresponding formula is the two-point Gaussian quadrature formula. Hence the family (3.4) also contains a multivariate analogue of a Gaussian quadrature formula. It may be mentioned that the case $\lambda=1 / \sqrt{d+2}$ of (3.4) is included in [16, p. 307, formula $T_{n}: 2-1$ ] where it is highlighted as a very useful formula.

For the sake of completeness we also present an error bound for the formula (3.4) when $\lambda \in\left(\lambda_{d}^{*}, 1\right)$. This estimate is not sharp but it becomes sharp at the boundary points $\lambda=\lambda_{d}^{*}$ and $\lambda=1$.

Proposition 6.4. Let $\mathcal{S} \subset \mathbb{R}^{d}$ be a non-degenerate simplex with vertices $\boldsymbol{x}_{0}, \ldots, \boldsymbol{x}_{d}$ and center of gravity $\boldsymbol{x}^{*}$. Let $f: \mathcal{S} \rightarrow \mathbb{R}$ be twice continuously differentiable. Then, for $\lambda \in\left(\lambda_{d}^{*}, 1\right)$, the remainder of the formula (3.4) satisfies

$$
\left|R_{d}(\lambda)[f]\right| \leq\left[\frac{1-\lambda^{2}(d+2)}{(d+1)(d+2)}+\frac{2\left(\lambda-\lambda_{d}^{*}\right)}{\left(1-\lambda_{d}^{*}\right)(d+2)}\right] \frac{\left|f^{\prime \prime}\right|}{2} \sum_{i=0}^{d}\left\|\boldsymbol{x}_{i}-\boldsymbol{x}^{*}\right\|^{2} .
$$

Lemma 6.1 also allows us to establish sharp error bounds for the family (4.2) when $\alpha$ is in the range which admits an upper Hermite-Hadamard inequality.

Corollary 6.5. Let $\mathcal{S} \subset \mathbb{R}^{d}$ be a non-degenerate simplex with vertices $\boldsymbol{x}_{0}, \ldots, \boldsymbol{x}_{d}$ and center of gravity $\boldsymbol{x}^{*}$. Let $f: \mathcal{S} \rightarrow \mathbb{R}$ be twice continuously differentiable. Then for formulae (4.2) the error estimate

$$
\left|R_{d}^{*}(\alpha)[f]\right| \leq \frac{\left|f^{\prime \prime}\right|}{2}\left(\frac{1}{d+2}-\frac{\alpha}{d+1}\right) \sum_{i=0}^{d}\left\|\boldsymbol{x}_{i}-\boldsymbol{x}^{*}\right\|^{2},
$$

for $0 \leq \alpha \leq 1 /(d+1)$, holds. This estimate is sharp. Equality is attained for every function of the form 6.3 .

For $d=1$, we obtain error estimates for the family (1.4). In particular, we find that

$$
\left|R^{*}\left(\frac{1}{2}\right)[f]\right| \leq \frac{(b-a)^{2}}{48} \max _{a \leq x \leq b}\left|f^{\prime \prime}(x)\right|,
$$

which is a result proved in [6, p. 44, Theorem 34]. 
For the sake of completeness, we also present an error estimate for the formula (4.2) when $\alpha \in(1 /(d+1), 1)$. This estimate is not sharp, but it becomes sharp at the boundary points $\alpha=1 /(d+1)$ and $\alpha=1$.

Proposition 6.6. Let $\mathcal{S} \subset \mathbb{R}^{d}$ be a non-degenerate simplex with vertices $\boldsymbol{x}_{0}, \ldots, \boldsymbol{x}_{d}$ and center of gravity $\boldsymbol{x}^{*}$. Let $f: \mathcal{S} \rightarrow \mathbb{R}$ be twice continuously differentiable. Then, for $\alpha \in(1 /(d+1), 1)$, the remainder of the formula (4.2) satisfies

$$
\left|R_{d}^{*}(\alpha)[f]\right| \leq \frac{d+(1-\alpha)\left(d^{2}-2\right)}{d(d+1)(d+2)} \cdot \frac{\left|f^{\prime \prime}\right|}{2} \sum_{i=0}^{d}\left\|\boldsymbol{x}_{i}-\boldsymbol{x}^{*}\right\|^{2} .
$$

\section{Proofs}

Proof of Theorem 2.1. The affine function $L_{\boldsymbol{x}^{*}}$, specified in Proposition A, may be written as

$$
L_{\boldsymbol{x}^{*}}(\boldsymbol{x})=f\left(\boldsymbol{x}^{*}\right)+\left\langle\boldsymbol{c}, \boldsymbol{x}-\boldsymbol{x}^{*}\right\rangle
$$

for some $\boldsymbol{c} \in \mathbb{R}^{d}$. Since $L_{\boldsymbol{x}^{*}}(\boldsymbol{x}) \leq f(\boldsymbol{x})$ for $\boldsymbol{x} \in \mathcal{D}$, integration of both sides yields

$$
f\left(\boldsymbol{x}^{*}\right)|\mathcal{D}|+\left\langle\boldsymbol{c}, \int_{\mathcal{D}} \boldsymbol{x} \mathrm{d} \boldsymbol{x}-\boldsymbol{x}^{*}|\mathcal{D}|\right\rangle \leq \int_{\mathcal{D}} f(\boldsymbol{x}) \mathrm{d} \boldsymbol{x} .
$$

Taking the definition of $\boldsymbol{x}^{*}$ into account, we readily obtain (2.1). The case of equality is easily verified.

Now assume that (2.1) also holds with $\boldsymbol{x}^{*}$ replaced by $\boldsymbol{y}$ on the left-hand side. Then, in both inequalities, the equality sign will occur for affine functions, as we pointed out in the Introduction. Thus, for the affine function

$$
L: \boldsymbol{x} \longmapsto\left\langle\boldsymbol{y}-\boldsymbol{x}^{*}, \boldsymbol{x}-\boldsymbol{x}^{*}\right\rangle,
$$

we obtain $\left\|\boldsymbol{y}-\boldsymbol{x}^{*}\right\|=0$, and so $\boldsymbol{y}=\boldsymbol{x}^{*}$.

Proof of Theorem 2.2. Given the simplex $\mathcal{S}$ as in the theorem, there exist uniquely determined affine functions $L_{0}, \ldots, L_{d}$ satisfying

$$
\begin{aligned}
L_{i}(\boldsymbol{x}) & \geq 0 \quad \text { for } \boldsymbol{x} \in \mathcal{S}, i=0, \ldots, d, \\
\sum_{i=0}^{d} L_{i}(\boldsymbol{x}) & =1 \quad \text { for all } \boldsymbol{x} \in \mathbb{R}^{d},
\end{aligned}
$$

and

$$
\boldsymbol{x}=\sum_{i=0}^{d} L_{i}(\boldsymbol{x}) \boldsymbol{x}_{i} \quad \text { for all } \boldsymbol{x} \in \mathbb{R}^{d} .
$$

The numbers $L_{0}(\boldsymbol{x}), \ldots, L_{d}(\boldsymbol{x})$ are called the affine or barycentric coordinates of $\boldsymbol{x}$ with respect to the vertices of $\mathcal{S}$; see, e.g., [11, pp. 132-135]. Using Kronecker's delta, we have

$$
L_{i}\left(\boldsymbol{x}_{j}\right)=\delta_{i j}
$$

as a consequence of (7.3). 
From (7.1) and (7.2), we see that the right-hand side of (7.3) is a convex combination of $\boldsymbol{x}_{0}, \ldots, \boldsymbol{x}_{d}$ when $\boldsymbol{x} \in \mathcal{S}$. Hence, applying $f$ on both sides of (7.3) and making use of the convexity, we find that

$$
f(\boldsymbol{x}) \leq \sum_{i=0}^{d} L_{i}(\boldsymbol{x}) f\left(\boldsymbol{x}_{i}\right) .
$$

Since $L_{i}$ is an affine function for each $i$, the center-of-gravity rule is exact for the right-hand side of (7.5), and so integration of both sides gives

$$
\int_{\mathcal{S}} f(\boldsymbol{x}) \mathrm{d} \boldsymbol{x} \leq|\mathcal{S}| \sum_{i=0}^{d} L_{i}\left(\boldsymbol{x}^{*}\right) f\left(\boldsymbol{x}_{i}\right)
$$

where $\boldsymbol{x}^{*}$ is the center of gravity of $\mathcal{S}$. For a simplex it is known that the center of gravity is equal to the centroid, or equivalently,

$$
\boldsymbol{x}^{*}=\frac{1}{d+1} \sum_{j=0}^{d} \boldsymbol{x}_{j}
$$

Therefore,

$$
L_{i}\left(\boldsymbol{x}^{*}\right)=\frac{1}{d+1} \sum_{j=0}^{d} L_{i}\left(\boldsymbol{x}_{j}\right)=\frac{L_{i}\left(\boldsymbol{x}_{i}\right)}{d+1}=\frac{1}{d+1}, \quad i=0, \ldots, d .
$$

This completes the proof of (2.3) .

It remains to discuss the occurrence of equality. As we mentioned in the Introduction, every Hermite-Hadamard inequality yields equality for affine functions. To complete the proof, we have to show that in (2.3) there are no other cases of equality. For this, we note that, in dependence on $\boldsymbol{x}$, the right-hand side of (7.5) is the uniquely determined affine function which coincides with $f$ at the vertices of $\mathcal{S}$. Hence, if $f$ is a convex but not an affine function, then (7.5) must be strict for some $\widetilde{\boldsymbol{x}} \in \mathcal{S}$. Since $f$ is upper semicontinuous on $\mathcal{S}$ (see [13, p. $96, \mathrm{~K}(3)]$ ), the inequality (7.5) is strict in some neighborhood of $\widetilde{\boldsymbol{x}}$. This implies that the inequality (2.3) is strict.

Proof of Theorem [2.3. Let $\mathcal{K}^{*}$ be the convex hull of $\boldsymbol{x}_{0}, \ldots, \boldsymbol{x}_{n}$. Assume toward a contradiction that there exists a point $\widetilde{\boldsymbol{x}} \in \mathcal{K} \backslash \mathcal{K}^{*}$. Then, by a fundamental result on convex sets (see [13] p. 81, Theorem A]), there exists a hyperplane $L(\boldsymbol{x})=0$ that separates $\widetilde{\boldsymbol{x}}$ and $\mathcal{K}^{*}$. It may be normalized such that $L(\widetilde{\boldsymbol{x}})=1$. Then $L(\boldsymbol{x})<0$ for all $\boldsymbol{x} \in \mathcal{K}^{*}$. Consider now the function $f$ defined by

$$
f(\boldsymbol{x}):=L(\boldsymbol{x})_{+}:=\max \{L(\boldsymbol{x}), 0\} .
$$

It has the following properties:

(a) $f$ is continuous;

(b) $f$ is convex since it is the maximum of two convex functions;

(c) $f(\boldsymbol{x}) \geq 0$ for $\boldsymbol{x} \in \mathcal{K}$;

(d) $f(\boldsymbol{x})=0$ for $\boldsymbol{x} \in \mathcal{K}^{*}$;

(e) $f(\widetilde{\boldsymbol{x}})=1$.

Because of (a) and (e), there is a neighborhood of $\widetilde{\boldsymbol{x}}$ where $f$ is strictly positive, and so, in conjunction with (c), we find that $\int_{\mathcal{K}} f(\boldsymbol{x}) \mathrm{d} \boldsymbol{x}>0$. On the other hand, it 
follows from (d) that $\sum_{\nu=0}^{n} A_{\nu} f\left(\boldsymbol{x}_{\nu}\right)=0$. This contradicts 2.5). Hence, we must have $\mathcal{K}=\mathcal{K}^{*}$, and so statement (i) is verified.

If $n$ were less than $d$, then the points $\boldsymbol{x}_{0}, \ldots, \boldsymbol{x}_{n}$ would lie on a hyperplane, and so $\mathcal{K}$ could not have positive measure in $\mathbb{R}^{d}$. This proves statement (ii).

If $\boldsymbol{x}_{j}$ is an extremal point, then it can be separated from all the other points $\boldsymbol{x}_{\nu}$ by a hyperplane $L(\boldsymbol{x})=0$ such that $L\left(\boldsymbol{x}_{j}\right)=1$. Then, for the convex function $f: \boldsymbol{x} \mapsto L(\boldsymbol{x})_{+}$, we have $f\left(\boldsymbol{x}_{\nu}\right)=\delta_{j \nu}$ using Kronecker's delta. Hence the righthand side of (2.5) is equal to $A_{j}$ while the integral of $f$ is positive. This completes the proof of statement (iii).

If $n=d$, then, by what we have proved already, $\mathcal{K}$ is a non-degenerate simplex. Integrating the associated affine functions $L_{0}, \ldots, L_{d}$ which define the barycentric coordinates (see the proof of Theorem 2.2) with the help of the formulae of Theorems 2.2 and 2.3, and recalling (7.4), we find that $A_{j}=1 /(d+1)$ for $j=0, \ldots, d$. This completes the proof of statement (iv).

Proof of Theorem 2.4. As we pointed out in the Introduction, equality must occur in (2.7) throughout when $f$ is an affine function. In particular, for $f=1$, we find that $\sum_{\mu=0}^{m} A_{\mu}=1$. Hence

$$
\boldsymbol{y}:=\sum_{\mu=0}^{m} A_{\mu} \boldsymbol{y}_{\mu}
$$

is a convex combination of $\boldsymbol{y}_{0}, \ldots, \boldsymbol{y}_{m}$. Therefore, the convexity of $f$ implies that

$$
f(\boldsymbol{y}) \leq \sum_{\mu=0}^{m} A_{\mu} f\left(\boldsymbol{y}_{\mu}\right),
$$

and so

$$
f(\boldsymbol{y}) \leq \frac{1}{|\mathcal{S}|} \int_{\mathcal{S}} f(\boldsymbol{x}) \mathrm{d} \boldsymbol{x}
$$

However, by Theorem 2.1, this is only possible for $\boldsymbol{y}=\boldsymbol{x}^{*}$. Hence (2.8) holds.

Clearly, the points $\boldsymbol{z}_{0}, \ldots, \boldsymbol{z}_{n}$ are a convex combination of the vertices of $\mathcal{S}$, that is,

$$
\boldsymbol{z}_{\nu}=\sum_{i=0}^{d} \lambda_{\nu i} \boldsymbol{x}_{i}, \quad \nu=0, \ldots, n,
$$

where $\lambda_{\nu i} \in[0,1]$ and $\sum_{i=0}^{d} \lambda_{\nu i}=1$ for $\nu=0, \ldots, n$. Hence, by the convexity of $f$,

$$
\begin{aligned}
\sum_{\nu=0}^{n} B_{\nu} f\left(\boldsymbol{z}_{\nu}\right) & =\sum_{\nu=0}^{n} B_{\nu} f\left(\sum_{i=0}^{d} \lambda_{\nu i} \boldsymbol{x}_{i}\right) \leq \sum_{\nu=0}^{n} B_{\nu} \sum_{i=0}^{d} \lambda_{\nu i} f\left(\boldsymbol{x}_{i}\right) \\
& =\sum_{i=0}^{d}\left(\sum_{\nu=0}^{n} B_{\nu} \lambda_{\nu i}\right) f\left(\boldsymbol{x}_{i}\right) .
\end{aligned}
$$

Now it follows from Theorem 2.3 (iv) that

$$
\sum_{\nu=0}^{n} B_{\nu} \lambda_{\nu i}=\frac{1}{d+1}
$$

and so (2.9) holds. 
Proof of Theorem [3.1. This proof is somewhat longer, and therefore we split it into several parts. Since the statements of Theorem 3.1 are invariant under an affine transformation, we want to suppose that $\mathcal{S}$ is a regular simplex with edges of length 1. This will simplify our considerations in Parts 2, 3, and 4.

Part 1. We show that the left-hand side of (3.2) is a non-decreasing function of $\lambda$, which obviously implies that if (3.2) holds for some $\widetilde{\lambda} \in(0,1)$, then it also holds for all $\lambda \in[0, \widetilde{\lambda}]$.

For this aim, we consider any $0 \leq \lambda \leq \widetilde{\lambda} \leq 1$ and express $\boldsymbol{x}_{i}(\lambda)$ in terms of $\boldsymbol{x}_{0}(\widetilde{\lambda}), \ldots, \boldsymbol{x}_{d}(\widetilde{\lambda})$. Using that

$$
\boldsymbol{x}^{*}=\frac{1}{d+1} \sum_{j=0}^{d} \boldsymbol{x}_{j}(\widetilde{\lambda})
$$

and combining (3.1) with the corresponding equation for $\tilde{\lambda}$ in such a way that $\boldsymbol{x}_{i}$ disappears, we find after a short calculation that

$$
\boldsymbol{x}_{i}(\lambda)=\frac{\lambda d+\widetilde{\lambda}}{(d+1) \tilde{\lambda}} \boldsymbol{x}_{i}(\widetilde{\lambda})+\frac{\tilde{\lambda}-\lambda}{(d+1) \tilde{\lambda}} \sum_{\substack{j=0 \\ j \neq i}}^{d} \boldsymbol{x}_{j}(\widetilde{\lambda}), \quad i=0, \ldots, d .
$$

Obviously, this representation of $\boldsymbol{x}_{i}(\lambda)$ is a convex combination of $\boldsymbol{x}_{0}(\widetilde{\lambda}), \ldots, \boldsymbol{x}_{d}(\widetilde{\lambda})$. Therefore, as a consequence of the convexity of $f$,

$$
f\left(\boldsymbol{x}_{i}(\lambda)\right) \leq \frac{\lambda d+\widetilde{\lambda}}{(d+1) \widetilde{\lambda}} f\left(\boldsymbol{x}_{i}(\widetilde{\lambda})\right)+\frac{\widetilde{\lambda}-\lambda}{(d+1) \widetilde{\lambda}} \sum_{\substack{j=0 \\ j \neq i}}^{d} f\left(\boldsymbol{x}_{j}(\widetilde{\lambda})\right), \quad i=0, \ldots, d
$$

Now, summation over $i$ implies that

$$
\sum_{i=0}^{d} f\left(\boldsymbol{x}_{i}(\lambda)\right) \leq \sum_{i=0}^{d} f\left(\boldsymbol{x}_{i}(\widetilde{\lambda})\right)
$$

which completes Part 1 of the proof.

Part 2. Take a vertex $\boldsymbol{x}_{i}$ of $\mathcal{S}$ and consider the centers of gravity of all the 1faces, 2 -faces, etc., that contain $\boldsymbol{x}_{i}$. These points together with $\boldsymbol{x}_{i}$ and $\boldsymbol{x}^{*}$ (the latter may be interpreted as the center of gravity of the $d$-face) define a convex polytope $\mathfrak{P}_{i}$. Since we have assumed $\mathcal{S}$ to be regular, the $d+1$ polytopes $\mathfrak{P}_{0}, \ldots, \mathfrak{P}_{d}$ are congruent and their union is $\mathcal{S}$. Moreover, the intersection of all of them is $\boldsymbol{x}^{*}$, and any two of them have no common interior point (see Figure 1). When in (3.1) the parameter $\lambda$ moves from 0 to 1 , then $\boldsymbol{x}_{i}(\lambda)$ moves along the symmetry axis of $\mathfrak{P}_{i}$ from the vertex $\boldsymbol{x}^{*}$ to $\boldsymbol{x}_{i}$. Clearly, there is a value of $\lambda$, call it $\lambda_{d}^{*}$, such that $\boldsymbol{x}_{i}\left(\lambda_{d}^{*}\right)$ is the center of gravity of $\mathfrak{P}_{i}$. Hence, by Theorem 2.1

$$
f\left(\boldsymbol{x}_{i}\left(\lambda_{d}^{*}\right)\right) \leq \frac{1}{\left|\mathfrak{P}_{i}\right|} \int_{\mathfrak{P}_{i}} f(\boldsymbol{x}) \mathrm{d} \boldsymbol{x}, \quad i=0, \ldots, d .
$$

Now, since $|\mathcal{S}|=(d+1)\left|\mathfrak{P}_{i}\right|$, summation over $i$ yields that (3.2) holds for $\lambda=\lambda_{d}^{*}$.

Part 3. Let $\lambda=\lambda_{d}^{*}+\varepsilon$. We shall show that for all sufficiently small positive $\varepsilon$ we can find a convex function $f$ for which (3.2) is false.

Consider a hyperplane $\mathcal{H}_{i}$ which is perpendicular to the symmetry axis of $\mathfrak{P}_{i}$ and which cuts off a small piece of $\mathfrak{P}_{i}$ containing $\boldsymbol{x}^{*}$. Denote by $\mathfrak{Q}_{i}$ the remaining part of $\mathfrak{P}_{i}$. Then the center of gravity of $\mathfrak{Q}_{i}$ lies on the symmetry axis of $\mathfrak{P}_{i}$, but 


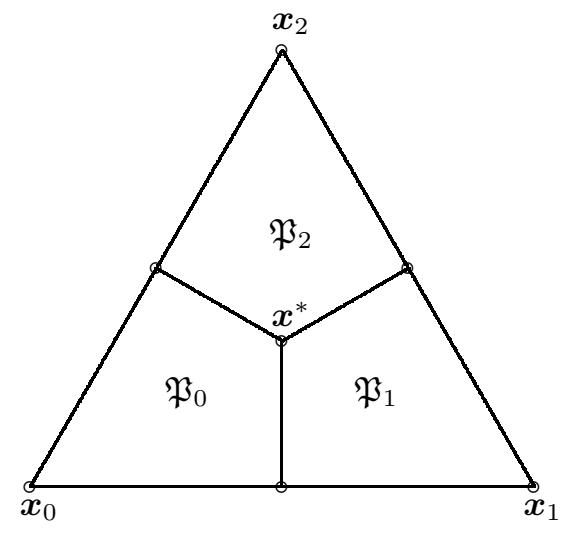

Figure 1. Decomposition of a regular $d$-dimensional simplex into $d+1$ congruent, convex polytopes. The case $d=2$.

it is somewhat closer to $\boldsymbol{x}_{i}$ than $\boldsymbol{x}_{i}\left(\lambda_{d}^{*}\right)$. By a continuity argument, it is clear that $\mathcal{H}_{i}$ can be chosen such that $\mathfrak{Q}_{i}$ has $\boldsymbol{x}_{i}(\lambda)$ as its center of gravity.

Now, for $i=0, \ldots, d$, let $L_{i}(\boldsymbol{x})=0$ be the equation of this hyperplane $\mathcal{H}_{i}$, normalized such that $L_{i}\left(\boldsymbol{x}_{i}\right)=1$, and define

$$
f(\boldsymbol{x}):=\max \left\{0, L_{0}(\boldsymbol{x}), \ldots, L_{d}(\boldsymbol{x})\right\} .
$$

Then $f$ is a convex function on $\mathcal{S}$ and

$$
f(\boldsymbol{x})=\left\{\begin{array}{cll}
L_{i}(\boldsymbol{x}) & \text { if } & \boldsymbol{x} \in \mathfrak{Q}_{i}, \\
0 & \text { if } & \boldsymbol{x} \in \mathfrak{P}_{i} \backslash \mathfrak{Q}_{i},
\end{array} \quad i=0, \ldots, d .\right.
$$

Hence, by Theorem 2.1

$$
\begin{aligned}
\int_{\mathfrak{P}_{i}} f(\boldsymbol{x}) \mathrm{d} \boldsymbol{x} & =\int_{\mathfrak{Q}_{i}} L_{i}(\boldsymbol{x}) \mathrm{d} \boldsymbol{x}=\left|\mathfrak{Q}_{i}\right| L_{i}\left(\boldsymbol{x}_{i}(\lambda)\right) \\
& <\left|\mathfrak{P}_{i}\right| f\left(\boldsymbol{x}_{i}(\lambda)\right)=\frac{|\mathcal{S}|}{d+1} f\left(\boldsymbol{x}_{i}(\lambda)\right),
\end{aligned}
$$

and so

$$
\frac{1}{|\mathcal{S}|} \int_{\mathcal{S}} f(\boldsymbol{x}) \mathrm{d} \boldsymbol{x}<\frac{1}{d+1} \sum_{i=0}^{d} f\left(\boldsymbol{x}_{i}(\lambda)\right),
$$

which shows that, in general, (3.2) is no longer true when $\lambda$ is a little larger than $\lambda_{d}^{*}$.

Part 4. Finally, we prove that $\lambda_{d}^{*}$ satisfies (3.3). For this, we determine the location of the center of gravity of $\mathfrak{P}_{i}$.

For fixed $i$, let us introduce a local, orthogonal coordinate system having the origin at $\boldsymbol{x}_{i}$ and the symmetry axis of $\mathfrak{P}_{i}$ as one of its axes, say, the $z$-axis, the orientation being chosen such that $\boldsymbol{x}^{*}$ has a positive $z$-coordinate.

First, we want to calculate the $z$-coordinates of the vertices of $\mathfrak{P}_{i}$. Consider a vertex which is the center of gravity of a $j$-face. Denote its distance from $\boldsymbol{x}_{i}$ by $a_{j}$ and its $z$-coordinate by $z_{j}$. Note that, by symmetry, $a_{j}$ (and also $z_{j}$ ) is the same for all the $j$-faces containing $\boldsymbol{x}_{i}$, and each $j$-face is a regular simplex in $\mathbb{R}^{j}$ with 


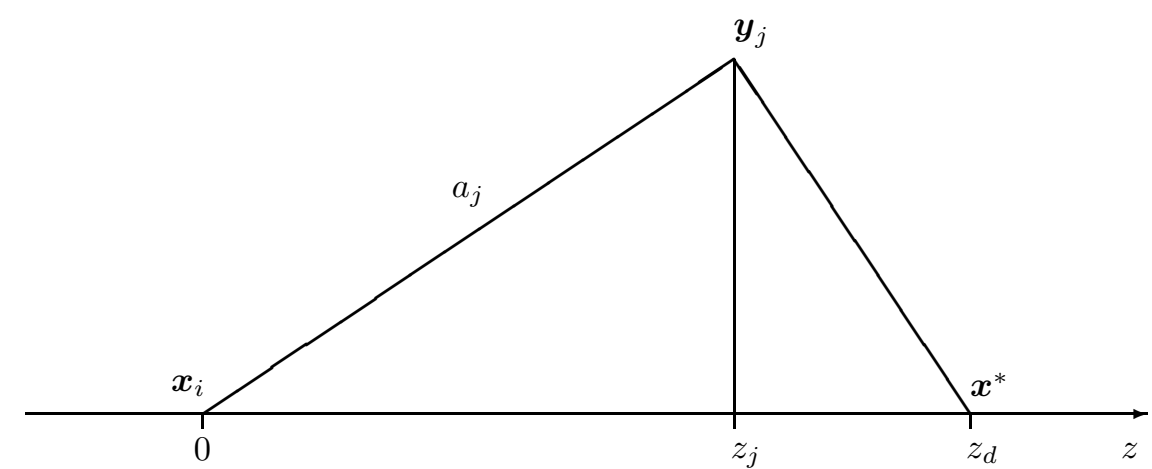

Figure 2. The local coordinate system. Here $\boldsymbol{y}_{j}$ is the center of gravity of a $j$-face containing $\boldsymbol{x}_{i}$.

edges of length 1 . If $h_{j}$ denotes the height of a $j$-face, then, by the Pythagorean theorem,

From these equations, we deduce that

$$
a_{j}^{2}+h_{j+1}^{2}=1 \quad \text { and } \quad h_{j+1}^{2}+\left(\frac{j}{j+1}\right)^{2} h_{j}^{2}=1 .
$$

$$
h_{j}=\sqrt{\frac{j+1}{2 j}} \quad \text { and } \quad a_{j}=\sqrt{\frac{j}{2(j+1)}}, \quad j=1, \ldots, d .
$$

Thus,

$$
z_{d}=a_{d}=\sqrt{\frac{d}{2(d+1)}} .
$$

From the underlying geometrical situation (see Figure 2), we deduce for the other vertices that

$$
z_{j} z_{d}=a_{j}^{2}, \quad j=1, \ldots, d-1
$$

Now a short reflection shows that $\mathfrak{P}_{i}$ can be decomposed into congruent simplices located around the $z$-axis, each simplex having the following vertices: $\boldsymbol{x}_{i}$, the center of gravity of a 1-face, the center of gravity of a 2 -face, ..., the center of gravity of a $(d-1)$-face, and $\boldsymbol{x}^{*}$. Because of symmetry, the $z$-coordinate $z^{*}$ of the center of gravity of $\mathfrak{P}_{i}$ is the same as the $z$-coordinate of the center of gravity of any of these simplices. Since, for a simplex, the coordinates of the center of gravity are the mean values of the corresponding coordinates of the vertices, we obtain

$$
\begin{aligned}
z^{*} & =\frac{1}{d+1} \sum_{j=1}^{d} z_{j}=\frac{1}{d+1} \sum_{j=1}^{d} \frac{a_{j}^{2}}{z_{d}} \\
& =\frac{1}{\sqrt{2 d(d+1)}} \sum_{j=1}^{d} \frac{j}{j+1}=z_{d}\left(1-\frac{1}{d} \sum_{j=2}^{d+1} \frac{1}{j}\right) .
\end{aligned}
$$

On the other hand, the representation (3.1) implies for our present coordinate system that

and so (3.3) follows.

$$
z^{*}=\lambda_{d}^{*} 0+\left(1-\lambda_{d}^{*}\right) z_{d}
$$


The proof is completed by combining the results of Parts 1 to 4 .

Proof of Corollary [3.2, Let $f$ be convex on $\mathcal{S}$. Then (see [13 p. 96, Problem K]) there exists a function $\bar{f}$ which is convex and continuous on $\mathcal{S}$ such that $\bar{f}(\boldsymbol{x})=f(\boldsymbol{x})$ when $\boldsymbol{x}$ is an interior point of $\mathcal{S}$ and $\bar{f}(\boldsymbol{x}) \leq f(\boldsymbol{x})$ when $\boldsymbol{x}$ is a boundary point of $\mathcal{S}$.

Let us now introduce

$$
E(f, \lambda):=\frac{1}{|\mathcal{S}|} \int_{\mathcal{S}} f(\boldsymbol{x}) \mathrm{d} \boldsymbol{x}-\frac{1}{d+1} \sum_{i=0}^{d} f\left(\boldsymbol{x}_{i}(\lambda)\right) .
$$

Then $E(\bar{f}, \cdot)$ is a continuous function on $[0,1]$ and $E(\bar{f}, \lambda)=E(f, \lambda)$ for $\lambda \in[0,1)$.

If $\bar{f}$ is not an affine function, then, by Theorem 2.2$] E(\bar{f}, 1)<0$ and, by Theorem $3.1 E\left(\bar{f}, \lambda_{d}^{*}\right) \geq 0$. Hence, by the intermediate-value theorem, there exists a $\widetilde{\lambda} \in\left[\lambda_{d}^{*}, 1\right)$ such that $E(f, \tilde{\lambda})=E(\bar{f}, \widetilde{\lambda})=0$. But, if $\bar{f}$ is an affine function, then $E(f, \lambda)=E(\bar{f}, \lambda)=0$ for all $\lambda \in[0,1)$. Thus, in any case, (3.5) is true.

Proof of Theorem 4.1. For $i=0, \ldots, d$, let $\mathcal{S}_{i}$ be the simplex whose vertices are $\boldsymbol{x}^{*}$ and all the vertices of $\mathcal{S}$ except for $\boldsymbol{x}_{i}$. These simplices decompose $\mathcal{S}$ and

$$
\left|\mathcal{S}_{0}\right|=\cdots=\left|\mathcal{S}_{d}\right|=\frac{|\mathcal{S}|}{d+1} .
$$

This is obvious for a regular simplex, but since an affine mapping maps the center of gravity of a simplex $\mathcal{S}$ to the center of gravity of the image of $\mathcal{S}$ and preserves the ratio of volumes, we conclude that (7.8) also holds for an arbitrary simplex $\mathcal{S}$.

Now, using Theorem 2.2 for the simplex $\mathcal{S}_{i}$, we find that, for every convex function $f$,

$$
\frac{1}{\left|\mathcal{S}_{i}\right|} \int_{\mathcal{S}_{i}} f(\boldsymbol{x}) \mathrm{d} \boldsymbol{x} \leq \frac{1}{d+1}\left[f\left(\boldsymbol{x}^{*}\right)-f\left(\boldsymbol{x}_{i}\right)+\sum_{j=0}^{d} f\left(\boldsymbol{x}_{j}\right)\right] .
$$

Adding these inequalities for $i=0, \ldots, d$, we obtain

$$
\frac{d+1}{|\mathcal{S}|} \int_{\mathcal{S}} f(\boldsymbol{x}) \mathrm{d} \boldsymbol{x} \leq f\left(\boldsymbol{x}^{*}\right)+\frac{d}{d+1} \sum_{j=0}^{d} f\left(\boldsymbol{x}_{j}\right),
$$

which shows that (4.1) holds for $\alpha=1 /(d+1)$. Since

$$
f\left(\boldsymbol{x}^{*}\right) \leq \frac{1}{d+1} \sum_{j=0}^{d} f\left(\boldsymbol{x}_{j}\right),
$$

as we know from Theorems 2.1 and 2.2, it follows that the right-hand side of (4.1) is a non-increasing function of $\alpha$ and that (4.1) holds for all $\alpha \in[0,1 /(d+1)]$.

The inequality (7.11) is strict unless $f$ is an affine function. In order to complete the proof, it therefore suffices to find a convex function $f$, different from an affine function, for which equality occurs in (4.1) when $\alpha=1 /(d+1)$. Such a function can be constructed as follows.

Let $L_{0}, \ldots, L_{d}$ be the affine functions describing the barycentric coordinates with respect to the vertices of $\mathcal{S}$, as introduced in the proof of Theorem 2.2, and define

$$
f(\boldsymbol{x}):=\max \left\{-L_{0}(\boldsymbol{x}), \ldots,-L_{d}(\boldsymbol{x})\right\}, \quad \boldsymbol{x} \in \mathcal{S} .
$$

Clearly, $f$ is convex on $\mathcal{S}$ and is not an affine function. Furthermore, for each index $j$ different from $i$, the difference $L_{j}(\boldsymbol{x})-L_{i}(\boldsymbol{x})$ is non-negative on $\mathcal{S}_{i}$ since, because 
of (7.4) and (7.7), it vanishes at all the vertices of $\mathcal{S}_{i}$ except for $\boldsymbol{x}_{j}$, where it attains the value 1. Hence $f(\boldsymbol{x})=-L_{i}(\boldsymbol{x})$ for $\boldsymbol{x} \in \mathcal{S}_{i}$. Thus, the restriction of $f$ to $\mathcal{S}_{i}$ is an affine function. By Theorem 2.2, this implies that equality occurs in (7.9) for each $i \in\{0, \ldots, d\}$ and, consequently, equality holds in (7.10), which is the case where $\alpha=1 /(d+1)$ in (4.1).

Proof of Theorem 5.1. Every polynomial of degree two in $d$ variables can be written as

$$
L(\boldsymbol{x})+\boldsymbol{x}^{\top} A \boldsymbol{x},
$$

where $L$ is an affine function and $A$ is a real, symmetric $d \times d$ matrix. Since the center-of-gravity rule and the vertices-of-a-simplex rule are exact for affine functions, it is enough to verify that $R_{d}^{\mathrm{Si}}[f]=0$ for

$$
f(\boldsymbol{x}):=\boldsymbol{x}^{\top} A \boldsymbol{x} .
$$

Using an integration formula of order three given in [16, p. 307, formula $T_{n}: 2-2$ ], we can express the integral of $f$ as

$$
\begin{aligned}
\frac{1}{|\mathcal{S}|} \int_{\mathcal{S}} f(\boldsymbol{x}) \mathrm{d} \boldsymbol{x}= & \frac{2-d}{(d+2)(d+1)} \sum_{i=0}^{d} f\left(\boldsymbol{x}_{i}\right) \\
& +\frac{4}{(d+2)(d+1)} \sum_{0 \leq i<j \leq d} f\left(\boldsymbol{x}_{i j}\right),
\end{aligned}
$$

where $\boldsymbol{x}_{i j}=\frac{1}{2}\left(\boldsymbol{x}_{i}+\boldsymbol{x}_{j}\right)$. Since

$$
4 f\left(\boldsymbol{x}_{i j}\right)=\left(\boldsymbol{x}_{i}+\boldsymbol{x}_{j}\right)^{\top} A\left(\boldsymbol{x}_{i}+\boldsymbol{x}_{j}\right)=f\left(\boldsymbol{x}_{i}\right)+2 \boldsymbol{x}_{i}^{\top} A \boldsymbol{x}_{j}+f\left(\boldsymbol{x}_{j}\right),
$$

we find for the second sum in (7.12) that

$$
\begin{aligned}
4 \sum_{0 \leq i<j \leq d} f\left(\boldsymbol{x}_{i j}\right) & =2\left[\sum_{i=0}^{d} \sum_{j=0}^{d} f\left(\boldsymbol{x}_{i j}\right)-\sum_{i=0}^{d} f\left(\boldsymbol{x}_{i}\right)\right] \\
& =(d-1) \sum_{i=0}^{d} f\left(\boldsymbol{x}_{i}\right)+\sum_{i=0}^{d} \sum_{j=0}^{d} \boldsymbol{x}_{i}^{\top} A \boldsymbol{x}_{j} \\
& =(d-1) \sum_{i=0}^{d} f\left(\boldsymbol{x}_{i}\right)+(d+1)^{2} f\left(\boldsymbol{x}^{*}\right) .
\end{aligned}
$$

Substituting this into (7.12), we arrive at

$$
\frac{1}{|\mathcal{S}|} \int_{\mathcal{S}} f(\boldsymbol{x}) \mathrm{d} \boldsymbol{x}=\frac{d+1}{d+2} f\left(\boldsymbol{x}^{*}\right)+\frac{1}{(d+2)(d+1)} \sum_{i=0}^{d} f\left(\boldsymbol{x}_{i}\right)
$$

This shows that $R_{d}^{\mathrm{Si}}[f]=0$.

Proof of Lemma 6.1. Consider the two functions

$$
g_{ \pm}:=\|\cdot-\boldsymbol{y}\|^{2} \frac{\left|f^{\prime \prime}\right|}{2} \pm f .
$$

For their Hessian matrix, we obtain

$$
H\left[g_{ \pm}\right](\boldsymbol{x})=\left|f^{\prime \prime}\right| I_{d} \pm H[f](\boldsymbol{x}),
$$


where $I_{d}$ is the $d \times d$ identity matrix. Recalling the definition of $\left|f^{\prime \prime}\right|$ in (6.1), we immediately see that $H\left[g_{ \pm}\right](\boldsymbol{x})$ is non-negative definite for all $\boldsymbol{x} \in \mathcal{S}$. Hence $g_{+}$ and $g_{-}$are both convex functions; see [13, p. 100, Theorem C]. Thus, if the given integration formula admits a lower Hermite-Hadamard inequality, then

$$
R_{d}\left[g_{+}\right] \geq 0 \quad \text { and } \quad R_{d}\left[g_{-}\right] \geq 0,
$$

which implies that

$$
-R_{d}\left[\|\cdot-\boldsymbol{y}\|^{2}\right] \frac{\left|f^{\prime \prime}\right|}{2} \leq R_{d}[f] \leq R_{d}\left[\|\cdot-\boldsymbol{y}\|^{2}\right] \frac{\left|f^{\prime \prime}\right|}{2} .
$$

This is equivalent to 6.2.

For an upper Hermite-Hadamard inequality, the proof is completely analogous.

As regards the statement on the occurrence of equality, it is enough to note that $\|\cdot-\boldsymbol{y}\|^{2}$ and $\|\cdot\|^{2}$ differ only by an affine function and that an integration formula is always exact for affine functions as soon as it admits a Hermite-Hadamard inequality.

Proof of Corollary 6.5. The desired results are obtained by using Lemma 6.1 in conjunction with Theorems 2.1, 2.2, and 3.1 respectively, choosing $\boldsymbol{y}=\boldsymbol{x}^{*}$, and calculating the integral of $\left\|\cdot-\boldsymbol{x}^{*}\right\|^{2}$ with the help of Theorem [5.1.

Proof of Proposition 6.4 Let us write $\boldsymbol{x}_{i}^{*}:=\boldsymbol{x}_{i}\left(\lambda_{d}^{*}\right)$ for $i=0, \ldots, d$. As is easily verified, the point $\boldsymbol{x}_{i}(\lambda)$, defined in (3.1), can be represented as

$$
\boldsymbol{x}_{i}(\lambda)=t \boldsymbol{x}_{i}+(1-t) \boldsymbol{x}_{i}^{*}, \quad \text { where } t=\frac{\lambda-\lambda_{d}^{*}}{1-\lambda_{d}^{*}} .
$$

From the theory of linear interpolation, we recall that, for a twice continuously differentiable function $\phi:[0,1] \rightarrow \mathbb{R}$, we have

$$
\phi(t)=(1-t) \phi(0)+t \phi(1)+\frac{t(t-1)}{2} \phi^{\prime \prime}(\theta)
$$

for some $\theta \in[0,1]$. Applying this result to

$$
\phi(t):=f\left(t \boldsymbol{x}_{i}+(1-t) \boldsymbol{x}_{i}^{*}\right)=f\left(\boldsymbol{x}_{i}(\lambda)\right),
$$

we find that

$$
f\left(\boldsymbol{x}_{i}(\lambda)\right)=(1-t) f\left(\boldsymbol{x}_{i}^{*}\right)+t f\left(\boldsymbol{x}_{i}\right)+\frac{t(t-1)}{2}\left(\boldsymbol{x}_{i}-\boldsymbol{x}_{i}^{*}\right)^{\top} H[f]\left(\boldsymbol{\xi}_{i}\right)\left(\boldsymbol{x}_{i}-\boldsymbol{x}_{i}^{*}\right),
$$

where $\boldsymbol{\xi}_{i}$ is an appropriate point on the line segment connecting $\boldsymbol{x}_{i}^{*}$ with $\boldsymbol{x}_{i}$. Using this representation, we obtain

$$
\begin{aligned}
R_{d}(\lambda)[f]= & \frac{1}{|\mathcal{S}|} \int_{\mathcal{S}} f(\boldsymbol{x}) \mathrm{d} \boldsymbol{x}-\frac{1}{d+1} \sum_{i=0}^{d} f\left(\boldsymbol{x}_{i}(\lambda)\right) \\
= & (1-t) R_{d}\left(\lambda_{d}^{*}\right)[f]+t R_{d}(1)[f] \\
& +\frac{t(t-1)}{2(d+1)} \sum_{i=0}^{d}\left(\boldsymbol{x}_{i}-\boldsymbol{x}_{i}^{*}\right)^{\top} H[f]\left(\boldsymbol{\xi}_{i}\right)\left(\boldsymbol{x}_{i}-\boldsymbol{x}_{i}^{*}\right) .
\end{aligned}
$$


Since $R_{d}(1)[f]=R_{d}^{\mathrm{vs}}[f]$, we can use (6.5), (6.6) for $\lambda=\lambda_{d}^{*}$, and (6.1) to conclude that

$$
\begin{aligned}
\left|R_{d}(\lambda)[f]\right| \leq & {\left[\frac{(1-t)\left[1-\left(\lambda_{d}^{*}\right)^{2}(d+2)\right]}{(d+1)(d+2)}+\frac{t}{d+2}+\frac{t(1-t)\left(1-\lambda_{d}^{*}\right)^{2}}{d+1}\right] } \\
& \times \frac{\left|f^{\prime \prime}\right|}{2} \sum_{i=0}^{d}\left\|\boldsymbol{x}_{i}-\boldsymbol{x}^{*}\right\|^{2} .
\end{aligned}
$$

Finally, expressing $t$ in terms of $\lambda$ according to (7.13), we arrive at the estimate of Proposition 6.4 by a straightforward calculation.

Proof of Corollary 6.5. In the situation of Theorem 4.1, we employ Lemma 6.1. choose $\boldsymbol{y}=\boldsymbol{x}^{*}$, and calculate the integral of $\left\|\cdot-\boldsymbol{x}^{*}\right\|^{2}$ with the help of Theorem 5.1

Proof of Proposition [6.6] We note that

$$
R_{d}^{*}(\alpha)[f]=\frac{(d+1) \alpha-1}{d} R_{d}^{\mathrm{cg}}[f]+\frac{(d+1)(1-\alpha)}{d} R_{d}^{*}\left(\frac{1}{d+1}\right)[f]
$$

and use the triangular inequality together with (6.4) and (6.7), setting $\alpha=1 /(d+1)$, for estimating the right-hand side.

\section{REFERENCES}

1. G. Allasia and C. Giordano, Approssimazioni bilaterali di integrali doppi di funzioni convesse, Calcolo, 20 (1983), 73-83. MR 86c:65025

2. H. Brass, Quadraturverfahren, Vandenhoeck \& Ruprecht, Göttingen, 1977. MR 56:1675

3. P.S. Bullen, Error estimates for some elementary quadrature rules, Publ. Elektroteh. Fak., Univ. Beogr., Ser. Mat. Fiz. 602-633, 97-103 (1978). MR 81h:65022

4. H. Busemann and G. C. Shephard, Convexity on nonconvex sets, Proceedings of the Colloquium on Convexity (Copenhagen, 1965) (W. Fenchel, ed.), Københavns Universitets Matematiske Institut, 1967, pp. 20-33. MR 36:4434

5. S.S. Dragomir, On Hadamard's inequality on a disk, J. Inequal. Pure Appl. Math. 1, Issue 1 (2000), Article 2, 11 p. MR 2001b:26023

6. S.S. Dragomir and C.E.M. Pearce, Selected Topics on Hermite-Hadamard Inequalities and Applications, Internet Publication, http://rgmia.vu.edu.au, 2000.

7. I. J. Good and R. A. Gaskins, The centroid method of numerical integration, Numer. Math. 16 (1971), 343-359. MR 44:1216

8. A. Guessab and G. Schmeisser, Sharp integral inequalities of the Hermite-Hadamard type, J. Approx. Theory 115 (2002), 260-288. MR 2003d:41028

9. P. C. Hammer, The midpoint method of numerical integration, Math. Mag. 31 (1958), 193195. MR 20:6191

10. F. B. Hildebrand, Introduction to Numerical Analysis, McGraw-Hill, New York, 1956. MR $17: 788 \mathrm{~d}$

11. P. J. Kelly and M. L. Weiss, Geometry and Convexity, John Wiley \& Sons, New York, 1979. MR 80h:52001

12. K. Petras, Quadrature theory of convex functions, Numerical Integration IV, ISNM Vol. 112 (H. Brass and G. Hämmerlin, eds.), Birkhäuser, Basel, 1993, pp. 315-329. MR 95c:65044

13. A. W. Roberts and D. E. Varnberg, Convex Sets, Academic Press, New York, 1973.

14. F. Stenger, Integration formulae based on the trapezoidal formula, J. Inst. Math. Appl. 12 (1973), 103-114. MR 52:2158

15. F. Stenger, Numerical Methods Based on Sinc and Analytic Functions, Springer-Verlag, New York, 1993. MR 94k:65003

16. A. H. Stroud, Approximate Calculation of Multiple Integrals, Prentice-Hall, Englewood Cliffs, N. J., 1971. MR 48:5348 
Department of Applied Mathematics, University of Pau, 64000 Pau, France

E-mail address: allal.guessab@univ-pau.fr

Mathematical Institute, University of Erlangen-Nuremberg, 91054 Erlangen, GerMANY

E-mail address: schmeisser@mi.uni-erlangen.de 